\title{
Analysis of Neurassthenia Effectiveness by Acupuncture Treatment
}

\author{
Huilian Shi
}

Qujing Hospital of Traditional Chinese Medicine, Qujing, 655000, China

\author{
Keywords: Neurassthenia, Treatment effectiveness, Acupuncture
}

\begin{abstract}
Objective: To analyze neurassthenia effectiveness by acupuncture treatment. Method: 100 cases of patients with neurasthenia were randomly divided into treatment group and control group according to random stratified sampling method. The patients in the control group were treated with routine anti-neurasthenia drugs, and the patients in the treatment group were treated with acupuncture and routine anti-neurasthenia drugs. Results: The total effective rate of the treatment group was $94.0 \%$, which was significantly higher than that of the control group (84.0\%). Conclusion: Routine ant neurological therapy combined with acupuncture and moxibustion is effective in the treatment of neurasthenia, which can improve the therapeutic effect of patients.
\end{abstract}

\section{Introduction}

Neurasthenia is a kind of neurosis. It is mainly excited by spirit, fatigue, irritability, irritability, tension, muscle tension and sleep disorders [1]. Modern Chinese medicine holds that mental illness is related to emotions, and also involves the functions of $\mathrm{Zang} \mathrm{Fu}$ and qi, blood, phlegm and fire depression. Qi, blood, phlegm and fire are both pathogenic and pathological products. Emotional disorder is Qi stagnation of blood phlegm and fire depression. The cause of seven excessive emotions leads to liver and gallbladder dysfunction, qi stagnation and phlegm, phlegm and Qi obstruction, liver, gallbladder and spleen disharmony, spleen and stomach ascension and descent and Qi depression. Initial pathogenesis of the disease begins in the liver, mainly Qi depression, resulting in liver depression, gallbladder heat, spleen suppression and less absorption, insufficient Qi and blood, heart loss and gradually fire, Yin and Yang Qi and blood loss holes. The initial disease is real, and the disease is in the liver, gallbladder and spleen. Blood addiction is endogenous, Yin Yang Qi blood disorder, phlegm coagulation and blood deficiency. Long illness is empty, and the disease is in the heart, spleen and kidney. Neurasthenia with neurasthenia and depression, under the condition of long-term anxiety, tension, anger and fear, can inhibit immune function, inhibit cell function, decrease immune function of macrophages, interfere with physiological function of viscera, promote immune function decline and aggravate neurosis. Over-intensity of stressors lasts for too long, which makes people with neurological genetic constitution and poor stress prone to anxiety and depression. In the situation of social competition, work and study stress, interpersonal tension, frustration and separation, it leads to neurasthenia. Among them, women are more than men, and those with lower social status and lower economic status are more obvious [2].

Neurasthenia is a common psychological disease in clinic. It is mostly seen in mental workers. It is a neurotic disorder. The main symptoms are mental excitement, mental fatigue, emotional irritability and insomnia. Some patients are accompanied by dizziness, headache, palpitation, shortness of breath, menstrual disorder and impotence. Neurasthenia is a temporary disorder of excitatory and inhibitory functions of the brain caused by long-term persistent over progress of brain nerve activity. Neurasthenia lasts for a long time and lasts for several months, which has seriously affected the study, work and life of patients. And long-term neurasthenia can cause the occurrence of mental illness, anxiety, depression, light birth and so on, endangering the safety of patients 'lives. Anti-anxiety, sedation and hypnosis are often used in clinical treatment, but the effect is general, and drug dependence and other complications will occur in long-term use. The author took fifty patients as the research object and adopts acupuncture and moxibustion treatment. The effect is analyzed as follows. 


\section{Materials and Method}

\subsection{Materials.}

One hundred patients with neurasthenia were randomly selected from April 2017 to June 2017. The diagnosis was in accordance with the diagnostic criteria of CCMD-2R neurasthenia: the patient's conscious memory decreased significantly or almost disappeared, and they could not work and study; they were depressed, dizzy, palpitation, insomnia and dreaminess, decreased attention, anxiety and depression. Neurasthenia caused by psychiatric disorders and personality splitting were excluded; no pregnant or lactating women; patients signed informed consent when they participated in the study. All patients were divided into treatment group and control group by random stratified sampling, 50 cases in each group, 26 males and 24 females in treatment group; age 20-54 years, average age (36.7 \pm 5.8 ) years; course of disease $6-10$ years, average course of disease (3.5 \pm 2.2 ) years; control group, 28 males and 22 females, age 20-56 years, average age (38.2 \pm 6.0$)$ years; course of disease 6-10 years, average course of disease with (3.8 \pm 2.1$)$ years. There was no significant difference in general data between the two groups $(\mathrm{P}>0.05)$.

\subsection{Method.}

General treatment: The treatment group was treated with acupuncture and moxibustion on the basis of routine anti-neurasthenia drugs. The control group was given conventional anti neurasthenia drugs only. Patients with depression were treated with Doxepin 25-50 mg orally every night, Naofukang $0.8 \mathrm{~g}$ orally, three times a day, to improve the function of brain cells; Shuledian $2 \mathrm{mg}$ orally before bedtime, if insomnia is serious, the dosage was increased to 4-6 mg. To minimize drug dependence, all drugs were discontinued after 2 weeks and reused after evaluation of therapeutic effect.

Acupuncture and Moxibustion: main points: choose Shen men and sleep two points. Dialectical allocation of acupoints: Yin deficiency and fire prosperity plus Taixi, Xinshu, Zusanli and Daling; Xinshu, Sanyinjiao, Pishu; phlegm heat internal disturbance plus Gongsun, Inner atrium, Fenglong; Liver depression and fire plus Foot Qiaoyin, Fengchi, Xingjian. With the matching points: forgetful, Baihui, Zhi room; many dreams plus soul households; dizziness plus Hegu, Yin Tang. I.23 Method: Those with Yin deficiency and fire prosperity should nourish Yin and Qianyang, only needle without moxibustion, placing reinforcing and reducing fire, and those with both heart and spleen deficiency should use both acupuncture and moxibustion to supplement heart and spleen; those with phlegm-heat internal disturbance should only be needled without moxibustion, and those with purging heat to remove phlegm and liver depression to remove fire should only be needled without moxibustion, using purging method to calm liver and reduce fire, seven times as a course of treatment 1 times every other day, after 2 courses of treatment, observe the therapeutic effect.

The criteria for judging the clinical efficacy of neurasthenia patients in this study are as follows: (1) healing, all clinical symptoms of patients disappear completely, and their sleep can be normal; (2) effective, the main clinical symptoms of patients have improved, and the quality of sleep has also improved; (3) ineffective, clinical symptoms of patients have not changed. Or worsening patients. The total effective rate of patients in clinical treatment is [(cured cases + effectual cases + effective cases] / total number of patients] *100.00\%.

The sleep quality of the two groups was $92.0 \%$ (46/50) in the treatment group and $78.0 \%$ (39/50) in the control group. The difference was statistically significant $(\mathrm{P}<0.05)$.

\section{Discussion}

Neurasthenia is a common neurological disorder in clinic. Its main manifestations are debilitating symptoms, excitement symptoms and emotional symptoms. Patients often suffer from mental retardation, inattention, active thinking, difficulty in directional thinking, annoyance, irritability, stiffness of neck, dizziness, sleep disorder, insomnia and multiple dreams. Repeated onset, prolonged course of disease, serious impact on the quality of life of patients, causing work and learning 
disabilities. In clinical treatment of neurasthenia, drugs are usually used to control the condition, which is difficult to achieve curative effect, and long-term drug dependence will occur in patients. And clinical medicines mostly use psychotropic drugs such as insomnia and depression, but the clinical efficacy is limited and the effect is not good. In traditional Chinese medicine, mental weakness belongs to the category of "palpitation" and "insomnia". The heart is the lesion site, with the mixture of deficiency and reality. The patient's body is damaged by emotional disturbance and viscera organs, resulting in disorder of Zang Fu organs and Qi and Yin, and imbalance of yin and Yang. And hurt the heart and liver, emotional disturbance, palpitation and chagrin; long-term insomnia will lead to inflammation of deficiency fire, chest tightness, headache and discomfort. Therefore, the disorder of Qi and Qi, the discord between Qi and blood are the common causes of neurasthenia. Therefore, acupuncture and moxibustion therapy can be used to adjust the meridian Qi and blood by stimulating acupoints, balance the body Yin and Yang, and restore the function of organs. Acupuncture and moxibustion mainly focus on Shenmen acupoint and sleeping acupoint, through Shenmen acupoint tranquilizing heart and tranquilizing mind, sleeping acupoint to improve sleep quality; and according to individual conditions of patients, dialectical implementation of acupoints, in order to make up for its deficiencies, play the role of yin-yang balance. In addition, acupuncture and moxibustion can balance the function of autonomic nerve, promote the EEG of neurasthenia patients to return to normal, effectively improve the clinical symptoms of patients, restore neurological symptoms of patients. In this study, the total effective rate of the treatment group was significantly higher than that of the control group, and the quality of grade I sleep was also higher than that of the control group $(\mathrm{P}<0.05)$.

Baihui and Si Shen Cong are located in the head and are actually one of the scalp needles. The author found that it was difficult for most insomniacs to insert needles into their scalp during the process of needling during $\mathrm{W}$ practice and oral follow-up in postgraduate stage, which was exaggerated to say that knife and gun could not penetrate. The tightrope state of scalp is positively correlated with the degree of insomnia. That is to say, the more serious the accident is, the longer the time is, the tighter the scalp is, and the tighter the degree of tightness of the patients whose sleep is not very serious is also on the low side. Once the needle is inserted, patients are easy to fall asleep in the process of needling, which is actually related to needling to relax the scalp. Therefore, the author believes that needling scalp needle can improve tension and then affect insomnia. Referring to the literature, no specific research has been found on the causes of improving insomnia. The scalp is like a piece of soil. If the scalp is in a tight rope for a long time, it is like the land about to crack. Acupuncture is like loosening soil. After that, oxygen or rain and sunshine can be received from nature. Therefore, acupuncturing Baihui and Sishensheng scalp needles can improve the tension of scalp and insomnia. The combination of Baihui and Sishencong acupuncture, whether directed toward Baihui or centered around Baihui and directed toward four directions, wraps around the whole top of the head, plays a role in regulating the whole brain, so as to nourish and calm the mind, and regulate the balance of Yin and Yang between the original spirit and the original spirit.

Liver disease and gallbladder, phlegm and fire disturbing the mind, one is frightened, upset, sleepless, impatient and restless. Cholera, phlegm and heat disturbing mind and mind are restless, dreamy and easy to wake up. Therefore, the phlegm in the gallbladder meridian does not belong to the cause of insomnia. Stagnation of the liver causes the spleen and stomach to move up and down. Chest pain and rest, irritated, chest fullness, abdominal pain. Liver depression and spleen depression can lead to splenic dysfunction, water-wet cohesion, phlegm turbidity, abdominal distension, difficulty in sleeping, early awakening nightmare, memory loss and fatigue.

Gallbladder stagnation and spleen and stomach produce phlegm, phlegm and heat. Stagnation of liver Qi leads to disharmony of lung function, depression and stagnation of phlegm. Stagnation of liver qi and disharmony of heart function, liver qi depression and heart qi deficiency, annoying and irritability, chest distress and restlessness. Liver depression is becoming more and more intense, causing distress, irritability, insomnia, more dreams, forgetfulness, dizziness, palpitation and anxiety. Traditional Chinese medicine believes that this disease belongs to insomnia, palpitation and other diseases, the location of the disease is in the heart, often manifested as a mixture of deficiency and 
reality. Most of them are due to emotional impairment, sadness, worry, or shock and heart, gallbladder and spleen, which lead to the disorder of viscera and qi, Yin and yang. If the heart and liver are injured, there will inevitably be emotional anxiety, palpitation and chagrin; insomnia for a long time, inflammation of deficient fire, chest tightness, headache discomfort and other symptoms. Therefore, the etiology of this disease is mostly caused by five extreme diseases, which leads to disorder of Qi and blood and disharmony between Qi and blood. Acupuncture and moxibustion therapy can adjust meridian qi and blood by stimulating acupoints, so as to achieve yin-yang balance and restore organ function. Neiguan is the collateral of the pericardium of the hand jueyin, which can regulate the upper and middle Jiao Qi machine to achieve the effect of broadening the chest, regulating Qi and tranquilizing the mind; Shenmen is the original point of the heart meridian, which can fill the essence, nourish the heart and calm the mind; Sleeping Point is the clinical effective point, which can nourish the mind, calm the mind and concentrate the mind. According to syndrome differentiation and specific symptoms and symptoms, acupoints are allocated to play the functions of catharsis and tonifying yin, balancing yin and yang, harmonizing Qi and blood, calming mind and calming mind. It is also necessary to cooperate with proper sports and psychological counseling when necessary.

To sum up, acupuncture and moxibustion treatment of neurasthenia can clear the heart and clear the mind, which conforms to the theory of "heart is the master of five Zang-organs and six fu-organs" in traditional Chinese medicine, and embodies the characteristics of syndrome differentiation and treatment of traditional Chinese medicine, and is worthy of clinical promotion. From the results, it can be seen that routine anti-neurasthenia drugs combined with acupuncture treatment can significantly improve the total effective rate of treatment, improve the mental symptoms of patients, and improve the quality of sleep of patients.

\section{References}

[1] Sun Peng. Neurassthenia Acupuncture Treatment Effectiveness Analysis [J]. Guide of China Medicine, 2014, 12(20): 63-64.

[2] Chen Guochao, Zhang Genming, Chen Yiqin, et al. Application and Development of Row Acupuncture Treatment in the Nervous System Diseases [J]. Journal of Liaoning University of Traditional Chinese Medicine, 2016, 18(4): 148-150.

[3] Chen Dong, Sun Yuanzheng. Treating 16 cases of nervous vomiting by acupuncture [J]. Clinical Journal of Chinese Medicine, 2016, 8(1): 44-45.

[4] Zhu Wenxian, Wang Jun, Wang Qunsong, et al. Effect of acupuncture on the autonomic nervous system in patients with major depressive disorder [J]. Lishizhen Medicine and Materia Medica Research, 2018, 29(2): 381-383. 